\title{
Particle interaction and coercivity for acicular particles
}

\author{
Ching-Ray Chang and Jyh-Pone Shyu \\ Department of Physics, National Taiwan University, Taipei, Taiwan
}

\begin{abstract}
The influence of the packing factor on coercivity for an assembly of particles is calculated. A rigorously theoretical explanation for the origin of empirical constants in the phenomenologically linear expression is given. The nonlinear coercivity dependence for a small packing factor is attributed to the nonuniform distribution of particles and the surface effects.
\end{abstract}

\section{Introduction}

Single domain acicular particles find application in magnetic recording and some permanent magnets. The particles are usually aggregated within the magnetic media and strong interaction fields arise between particles. Therefore, the coercivity $H_{\mathrm{c}}(P)$, where $P$ is the volume packing factor, will be lower than the $H_{c}(0)$ of an isolated particle. To clear the dependence of the coercivity of an assembly of elongated single domain particles is an old but difficult problem since complicated many-body effects are involved. The earliest theoretical expression was given by Néel [1], and is still the most widely quoted, which is

$H_{\mathrm{c}}(P)=H_{\mathrm{c}}(0)(1-P)$.

However, this equation does not always agree with experiments, and its derivation is not convinving enough [2]. Thus, a more useful phenomenological relationship was proposed [3],

$H_{\mathrm{c}}(P)=A+B(1-P)$.

This expression takes the linear interpolation between two limits, i.e., the coercivity of $H_{\mathrm{c}}(0)$ and $H_{\mathrm{c}}(1)$. Here $B$ equals the constant in the Néel's formula and the empirical constant $A$ has been attributed to the effect of magnetocrystalline anisotropy [2]. Except for the very small packing factor, eq. (2) is usually consistent with experimental data and has been used for many years. Although computer simulations also confirmed this phenomenological expression [3], rigorously theoretical explanation does not exist up to now. Especially, the nonlinear behavior of coercivity for small packing factor was never clearly explained.

In the present work, we use a mean field model [4]

Correspondence to: Dr. Ching-Ray Chang, Department of Physics, National Taiwan University, Taipei, Taiwan. Tel.: +886-2-363-0231, ext. 3161; telefax: +886-2-363-9984; E-mail: crchang@phyc1.phys.ntu.edu.tw. to calculate the average particle-particle interaction energy for each pair of particles from first principles, then we deduce the relationship between coercivity and packing density. We find that the empirical constants $A$ and $B$ in eq. (2) result from the shape and crystalline magnetic anisotropy of the sample and of the individual particles inside it. It is suggested that the nonlinear behavior of coercivity for small packing factor can be explained with the variation of particle volume and the surface effects.

\section{Theory}

Considering the total energy of an assembly of particles as a sum of Zeeman energy, crystalline anisotropy energy and magnetic self-energy,

$E_{\mathrm{T}}=E_{\mathrm{Z}}+E_{\mathrm{K}}+E_{\mathrm{D}}$

Here we assumed all the easy axis are collinear. The total crystalline anisotropy energy for the uniaxial case can be expressed as

$E_{\mathrm{K}}=\sum_{i=1}^{n} v_{i} K_{1} \sin ^{2} \theta_{i}$

where $K_{1}$ is the first anisotropy constant. The total self-energy

$$
\begin{aligned}
E_{D} & =-\frac{1}{2} \int_{\sum v_{i}} \boldsymbol{M} \cdot\left(\sum_{j=1}^{n} \boldsymbol{H}_{j}\right) \mathrm{d} v \\
& =\sum_{i=1}^{n} E_{i}^{\text {self }}+\frac{1}{2} \sum_{i \neq j} E_{i j}^{\mathrm{int}},
\end{aligned}
$$

where

$$
\begin{aligned}
& E_{i}^{\text {self }}=\frac{1}{2} \int_{v_{i}} \boldsymbol{M} \cdot \boldsymbol{N}_{i} M \mathrm{~d} v, \\
& E_{i j}^{\text {int }}=\int_{v_{i}} \boldsymbol{M} \cdot \boldsymbol{N}_{j} \boldsymbol{M} \mathrm{d} v .
\end{aligned}
$$


$\boldsymbol{N}_{i}$ is the demagnetization tensor of the $i$ th particle. For dilute enough system those particles are nearly mutually independent. Therefore, with the same external field all the magnetizations of particles shall be always along the same direction. The expectational value of particle-particle interaction energy [4] can be derived as

$\left\langle E_{i j}^{\mathrm{int}}\right\rangle \approx M \cdot \frac{1}{V}\left[\frac{V_{\mathrm{p}}^{2}}{n^{2}}-\frac{\delta^{2}}{4(n-1)}\right]\left[\boldsymbol{N}_{b}-\left\langle\boldsymbol{N}_{\mathrm{p}}\right\rangle\right] \cdot M$.

Here $V_{\mathrm{p}}$ is the total particle volume, and $\delta$ is the half-width of size variation. $\boldsymbol{N}_{\mathrm{b}}, \boldsymbol{N}_{\mathrm{p}}$ are the demagnetization tensors of the sample and of the individual particles. Substituting eqs. (4), (5) and (8) into eq. (3), we obtain

$$
\begin{aligned}
E_{\mathrm{T}}= & -P V M \cdot \boldsymbol{H}+P V K_{1} \sin ^{2} \theta+\frac{P V}{2} \boldsymbol{M} \cdot\left\langle\boldsymbol{N}_{\mathrm{p}}\right\rangle \boldsymbol{M} \\
& +\frac{P^{2} V}{2}\left[\frac{n-1}{n}-\frac{F^{2}}{4 n}\right] \boldsymbol{M} \cdot\left(\boldsymbol{N}_{\mathrm{b}}-\left\langle\boldsymbol{N}_{\mathrm{p}}\right\rangle\right) \cdot \boldsymbol{M},
\end{aligned}
$$

and the coercive force is

$$
\begin{aligned}
H_{c}(P)= & \left(\frac{2 K_{1}}{M}+H_{c b}\right) \\
& +[1-W(P, n)]\left(\left\langle H_{\mathrm{cp}}\right\rangle-H_{\mathrm{cb}}\right),
\end{aligned}
$$

where $P=V_{\mathrm{p}} / V$ is the packing factor, and $\left\langle H_{\mathrm{cp}}\right\rangle, H_{\mathrm{cb}}$ are the coercivity from shape anisotropy of individual particle and sample, respectively. $W(P, n)$ is a weighting function

$W(P, n)=P\left(\frac{n-1}{n}-\frac{F^{2}}{4 n}\right)$,

where $F=\delta /\langle v\rangle$ and $\langle v\rangle$ is the average particle size. By changing variable, eq. (11) can be rewritten as

$W(P, n)=P-\frac{\langle v\rangle}{V}\left(1+\frac{F^{2}}{4}\right)$.

\section{Discussions}

Unless the number of particles is very small, $W(P$, $n$ ) is approximately equal to $P$ (fig. 1 ). Therefore, from eq. (10) we know that the phenomenologic relation, eq. (2), can be obtained. However, the empirical constants $A$ and $B$ are not interpretated as before [2], we find that $A$ represents the contributions of the magne-

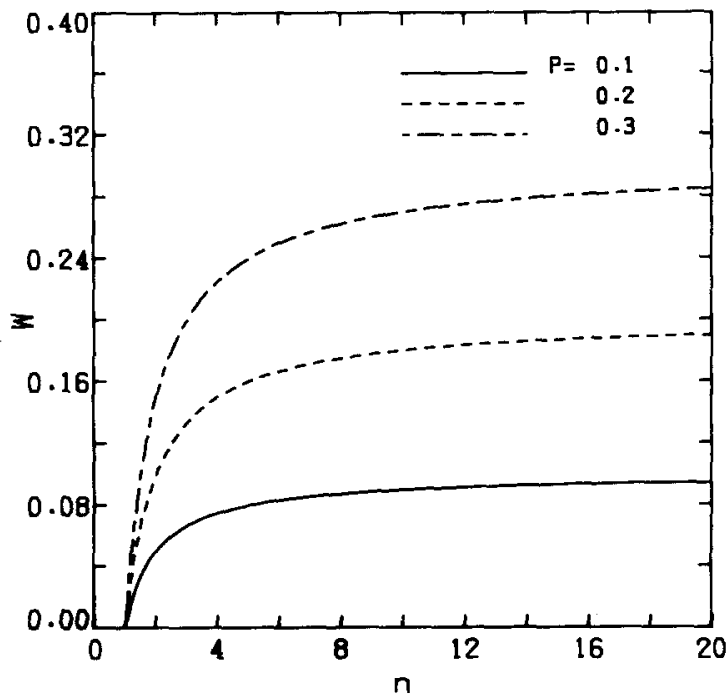

Fig. 1. The relationship of weighting function $W$ and $n$ for different $P$.

tocrystalline anisotropy of the particle and of the shape anisotropy of the sample, while $B$ is the part of the coercivity arising from the shape anisotropy of both the isolated particle and the sample. From eq. (10), we also know that Néel's formula is only valid when crystalline anisotropy does not exist and the shape of the sample is spherical. It is interesting to point out that $H_{c}(P)$ will be independent of $P$ for the same shape of the sample and the particle.

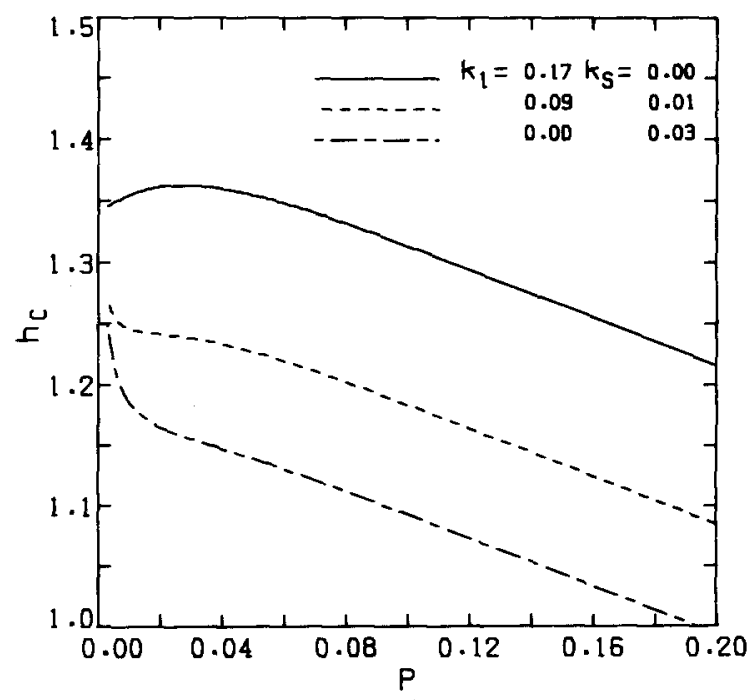

Fig. 2. Normalized coercivity versus $P$ for different $k_{1}$ and $k_{\mathrm{s}}$, where $k_{1}=K_{1} / M H_{\mathrm{cp}}, k_{\mathrm{s}}=K_{\mathrm{s}} / M H_{\mathrm{cp}} V_{\mathrm{c}}^{1 / 3}$ and $h_{\mathrm{c}}(P)=$ $H_{\mathrm{c}}(P) / H_{\mathrm{cp}}$. Here $F$ is 0.2 . 
For a very small packing factor, experimental data [3] show that the linear dependence of the coercivity for the packing factor does not hold. In order to understand the origin of this nonlinear behavior, several possible mechanisms for $P \ll 1$ are suggested as follows. With fixed average particle size, the number of particles should be proportionally reduced for a very small packing factor. Therefore particles cannot be treated as spreading over the sample, and the influence due to the shape of sample, i.e. the $\boldsymbol{N}_{\mathrm{b}}$ term in eq. (9), will be meaningless. Furthermore, these few particles may aggregate and then the assumption of uniform distribution fails; therefore, the dependence of coercivity for the packing factor can deviate from the prediction of linear behavior in eq. (11). In another way if one reduces the packing factor by reducing the particle size, it will induce a large surface to volume ratio for the particle and the surface effects probably dominate. In fact, the experimental process of dilution may change the average particle size and particle shape, especially for very dilute cases since too many times of scraping were done and the scraping processes are too long [5].

We can assume that all the particle aggregate into clusters for a very small packing factor and the clusters are separated from each other. Therefore, the coercivity of the sample is approximately equal to the coercivity of the cluster. With introducing a phenomenological surface term, the resultant coercivity can be modified as

$$
\begin{aligned}
H_{\mathrm{c}}(P)= & \left(\frac{2 K_{1}}{M}+H_{\mathrm{cc}}\right)+\left[1-P+\frac{\langle v\rangle}{V_{\mathrm{c}}}\left(1+\frac{F^{2}}{4}\right)\right] \\
& \times\left(H_{\mathrm{cp}}-H_{\mathrm{cc}}\right)+\frac{2 K_{\mathrm{s}}}{M\langle v\rangle^{1 / 3}}
\end{aligned}
$$

where $K_{\mathrm{s}}\langle v\rangle^{2 / 3}$ is the average surface energy of particles, $V_{c}$ is the volume of cluster and $H_{c c}$ is the demagnetizing field due to the shape of the cluster. In fig. 2, we also assume that the average particle size decreases with decreasing packing factor. The calculated results are shown in fig. 2 (different crystalline anisotropy constants are chosen for different curves here). The coercivity can be either increasing or decreasing for small packing factor of different surface anisotropy constant. It should be noticed that there is a very small bump for $P \ll 1$ at moderate value of $K_{\mathrm{s}}$ (fig. 2). This is because of a compromise between the decreasing of volume energy and the increasing of surface energy. Similar behavior were usually found in experimental data [3].

This work was supported by the National Science Council, Taiwan, under grant no. NSC-81-0208-M00232 .

\section{References}

[1] L. Néel, Compt. Rend. 234 (1947) 1150.

[2] J.E. Knowles, J. Magn. Magn. Mater. 25 (1981) 105.

[3] J.E. Knowles, IEEE Trans. Magn. MAG-21 (1985) 2576.

[4] C.-R. Chang and J.P. Shyu, J. Appl. Phys. 70 (1991) 7504.

[5] S. Umeki, H. Sugihara, Y. Taketomi and Y. Imaoka, IEEE Trans. Magn. MAG-17 (1981) 3014. 